\title{
Neue Wege in der wasserbezogenen Umweltsensorik
}

\author{
$\underline{\text { Michael Seidel }^{1}}$ \\ ${ }^{1}$ Lehrstuhl für Analytische Chemie und Wasserchemie, Technische Universität München, Deutschland
}

\section{Einleitung}

Mit diesem Beitrag soll eine Perspektive für den Einsatz von Sensoren in der Umweltanalytik dargestellt werden, welche sich dem Zukunftsthema Digitalisierung stellt. Die Digitalisierung ist in vielen Bereichen das Zukunftsthema und Sensorik wird mittlerweile ubiquitär eingesetzt. Aus diesem Grund ist es unumgänglich, diese Fortschritte in der Umweltanalytik nutzbar zu machen und eine wasserbezogene intelligente Umweltsensorik zu entwickeln. Umweltanalytik mit Sensoren bietet die Möglichkeit, definierte Parameter einfach, kostengünstig, an jedem Ort in kürzester Zeit und möglichst autark zu erfassen. Sensoren können der Schlüssel für ein effizientes Umweltmonitoring sein, um dynamische Prozesse frühzeitig erkennen und steuern zu können. Eine Vielzahl an physikalischen, chemischen und biochemischen (Bio-) Sensoren existieren. Die Vernetzung dieser Sensoren und die intelligente Auswertung möglichst in Echtzeit ist die Herausforderung für zukünftige Forschung in allen Bereichen der Umweltanalytik (siehe Abb. 1). Beispielhaft sind hier die wirkungsbezogene Analytik und die Prozesskontrolle zu nennen.

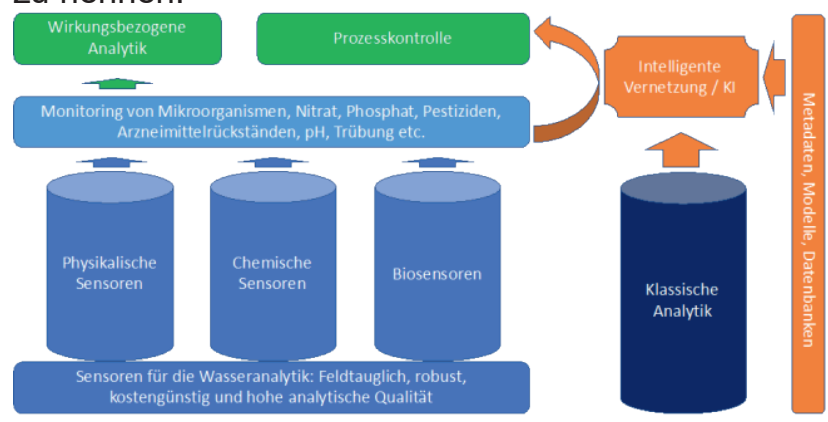

Abb. 1: Übersicht der Nutzung der vernetzten Sensorik in Verbindung mit Lernalgorithmen der $\mathrm{KI}$ und der klassischen Analytik für ein immerfortes Verbessern des Erlernten.

Mit einer Vielzahl von physikalischen, chemischen Sensoren oder Biosensoren werden zeitabhängige Messdaten erzeugt, welche eine künstliche Intelligenz (KI) nutzen kann, um mit Hilfe eines Lernalgorithmus komplexe Systeme zu steuern. Wie im Schulalltag ist aber eine enge Lehrer-SchülerBeziehung wichtig. In diesem Bild ist die klassische Analytik der Lehrer oder die Lehrerin, welche das in Übungen erarbeitete Ergebnis verifiziert. Diesen Prozess der Erkenntnisgewinnung und Überprüfung nennen wir Lernen. Nur in diesem Zusammenspiel kann eine $\mathrm{KI}$ effektiv für die Umweltanalytik genutzt werden. Komplexe Prozesse können mit KI sehr schnell erfasst und technische wasserführende Anlagen könnten in Zukunft autonom gesteuert werden.

\section{Anwendungsbeispiele für Kl-genutzte vernetzte Sensorik}

\section{Legionellenmonitoring}

In wassertechnischen Anlagen, wie zum Beispiel Verdunstungskühlanlagen oder Autowaschanlagen ist die Konditionierung des Wassers von großer Bedeutung. Ziel ist es, mit Hilfe von Bioziden und Korrosionsschutz, die Anlagen dauerhaft und sicher zu nutzen. Mittlerweile ist bekannt, dass in diesen Anlagen bei Temperaturen zwischen $45-65{ }^{\circ} \mathrm{C}$ sich Legionellen so vermehren können, dass sie eine Gefahr für den Menschen darstellen. Atmet ein Mensch die aus diesen Anlagen entstehenden Bioaerosole ein, so kann es zu einer sehr starken Lungenentzündung kommen, der LegionellenPneumonie. ${ }^{1}$ Der Gesetzgeber hat mit der 42. Bundesimmissionsschutzverordnung (42. BImSchV) die Betreiber von Verdunstungskühlanlagen, Kühltürmen und Nassabscheidern in die Pflicht genommen, die Konzentration an Legionellen unterhalb eines Maßnahmenwertes zu halten und bestenfalls die Legionellen in den Anlagen zu eliminieren. ${ }^{2}$ Diese Forderung kann zur Folge haben, dass eine betroffene Anlage erst einmal stillgelegt werden muss, was zu enorm hohen ökonomischen Verlusten führen kann. Da die Konzentration von Legionellen mit Kulturverfahren bestimmt werden muss und Legionellen sich nur sehr langsam vermehren lassen, erhält der Betreiber erst nach 12 Tagen ein Ergebnis. Neben der langen Wartezeiten sind falsch-negative Ergebnisse ein analytisches Problem. ${ }^{2}$ Bei positiven Befunden über 100 kolonienbildenden Einheiten (KBE) in $100 \mathrm{~mL}-10.000 \mathrm{KBE} / 100 \mathrm{~mL}$ (Prüfwert) ist der Betreiber verpflichtet, mit geeigneten Maßnahmen die Legionellenkonzentration zu reduzieren. Oberhalb des Maßnahmenwertes muss der Betreiber die Anlage der zuständigen Behörde melden. Wie kann hier die Sensorik genutzt werden? Die Aufgabe wäre es, dass durch intelligente Steuerung sehr gezielt in geringen Dosen chemische Wirkstoffe, wie zum Beispiel Biozide und Korrosionsschutz in wassertechnische Anlagen einzuleiten und durch eine sensorische Überwachung das Wachstum von Legionellen zu verhindern. Mit dieser Maßnahme könnte dem Betreiber geholfen werden, weil die Anlage ständig überwacht wird und das Risiko einer Legionellenvermehrung minimiert wird. Grundvoraussetzung für diesen Ansatz ist es aber, dass eine 
$\mathrm{KI}$ aufgebaut wird, die nicht nur mit sensorischen Messdaten gefüttert wird, sondern im Vorfeld das Zusammenspiel zwischen Wasserchemie und Legionellenvermehrung quantitativ erfasst wird.

Kontinuierliche Prozessüberwachung in Kläranlagen Die Abwasserbehandlung ist wichtig, damit eine umweltbelastende Emission von Schadstoffen in das Oberflächenwasser vermieden wird. Heutzutage wird das Spektrum an möglichen Kontaminationen auf Mikroschadstoffe und Pathogene erweitert, weil sie für den Menschen und dem Ökosystem gefährlich sind. Die biologische Abwasserbehandlung und zukünftig die 4. Reinigungsstufe sind für die Reduktion dieser Komponenten von großer Bedeutung. Eine effiziente biologische Abwasserbehandlung müsste aufwändig messtechnisch überwacht werden, damit die für Ammoniumoxidation und Denitrifizierung zuständigen Mikroorganismen optimal arbeiten können. ${ }^{3}$ Aus Kostengründen und die Umsetzbarkeit in die Praxis, werden nur einfache Summenparameter in regelmäßigen Abständen erfasst, mit denen der Kläranlagenmeister die Abwasserbehandlung überwacht. In Kläranlagen ließen sich Sensorsysteme für eine optimierte Prozesskontrolle einsetzen, wenn sie relevante Parameter, wie zum Beispiel gelöstes Ammonium, Nitrat, Phosphat, Sauerstoff, Gesamtheit der organischen Kohlenstoff (TOC) oder den chemischen Sauerstoffbedarf (CSB), sowie mikrobiologische Parameter wie die Keimzahl und allgemeine Parameter wie Temperatur, Trübung und Volumenstrom kontinuierlich erfassen können. Zielsetzung wäre es die Abwasserbehandlung so zu steuern, dass Mikroschadstoffe, antibiotikaresistente Bakterien oder Pathogene effizient reduziert werden, ohne diese bestimmen zu müssen. Damit wäre es mit Sensorik in Zusammenspiel mit der KI möglich, dass die Ökosysteme nachhaltig stabilisiert sind und sich eventuell sogar geklärtes Abwasser für den Wiedergebrauch mit hoher Sicherheit nutzen lässt. Weitere Anwendung der intelligenten Sensorik in der Abwasserbehandlung ist die Reduzierung von klimaschädlichen Gasen, wie Lachgas und Methan, welche in der biologischen Abwasserbehandlung entstehen können und aktuell unkontrolliert in die Umwelt gelangen. Hier ist neben der Überwachung der Leitparameter und der Mikrobiologie, gleichzeitig der Einsatz einer Gassensorik für Methan und Lachgas von Nöten.

\section{Wirkungsbezogene Analytik}

Ein weiteres Einsatzszenario ist die wirkungsbezogene Analytik, welche in der Ökosystemanalyse zum Einsatz kommt. Wirkungsbezogene Analytik ist eine Methode, bei der nicht einzelne Wirkstoffe, sondern biologische Effekte der Gesamtprobe in ausgewählten Zielsystemen nachzuweisen. ${ }^{4}$ Somit können in einem nicht-gerichteten (non-target) Ansatz, Substanzen entdeckt werden, welche mit einer toxikologischen Wirkung in Zusammenhang gebracht wer- den, ohne sie zuvor in Betracht gezogen zu haben. Da biologische Systeme für ein stetiges Wassermonitoring meistens sehr aufwendig zu handhaben sind, wäre es denkbar, dass eine Vernetzung mit Sensoren in Zusammenspiel mit der KI genutzt wird.

\section{Algenmonitoring im Oberflächenwasser}

Ein letztes Beispiel ist der Aufbau eines intelligenten Algenmonitoringsystems, das als Frühwarnsystem genutzt werden kann. Hier ist es wichtig, nicht nur zu erkennen, ob sich im Oberflächenwasser Algen gebildet haben, sondern ob diese Algen Toxine gebildet haben, welche eine Gefahr für Mensch und Tiere sein kann. Dieses Anwendungsbeispiel kommt aus der eigenen Forschung, welches konkret vernetzte Sensorik und KI nutzt. Zusammen mit der A.U.G. Signals Ltd. und der Hydroisotop $\mathrm{GmbH}$ arbeitet die TUM in einem AIF-ZIM-Projekt an einem intelligenten Algenmonitoringsystem, das zur Überwachung von Oberflächenwasser eingesetzt werden soll. Das Auftreten von Algenblüten tritt immer häufiger im Oberflächenwasser auf, weil Cyanobakterien anthropogene Veränderungen wie Eutrophierung der Gewässer oder Klimawandel für ihren eigenen Vorteil nutzen. Algenblüten sind nicht nur für den Tourismus schlecht, sie können sogar eine Gefahr für das Trinkwasser sein, wenn zum Beispiel Süßwasser-Lagunen genutzt werden müssen. Cyanobakterien bilden Toxine, welche für Tiere und Menschen gesundheitsschädlich sind. Es ist zum Beispiel bekannt, dass in Seen badende Hunde gestorben sind, weil diese zu viele Cyanotoxine aufgenommen haben. Ein Frühwarnsystem könnte in Echtzeit lokal Informationen über den Zustand eines Gewässers liefern und damit die Sicherheit für Menschen und Tiere erhöhen.

\section{Intelligentes Algenmonitoring}

Das TRITON-Wasser-Sensorsystem von AUG Signals besteht aus zwei Hauptkomponenten (Abb. 2).
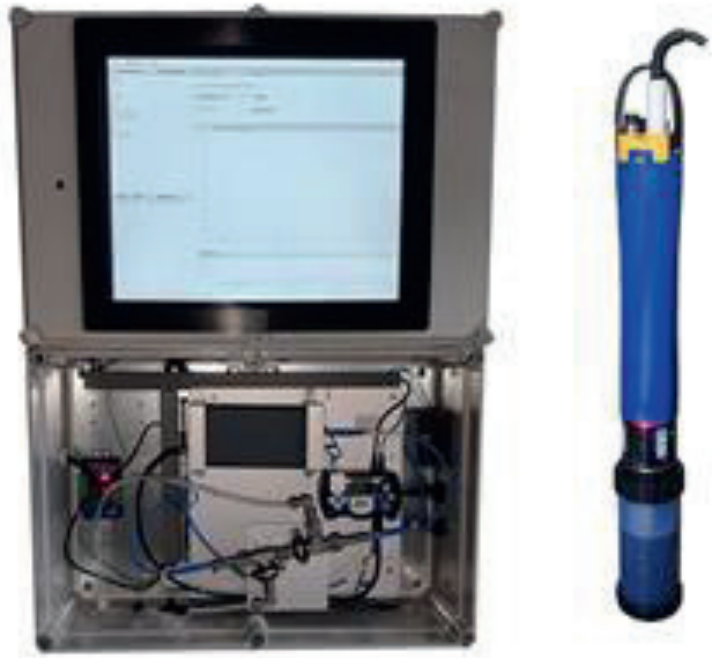
Abb. 2: Das TRITON-Wasser-Sensorsystem bestehend aus einem Durchflussmesssystem für die UVVIS-Absorptionsspektralanalyse, einer Multiparametermesssonde und ein Cloud-basiertes Datenmanagement-System.

Ein optisches Sensormodul liefert eine kontinuierliche UV-VIS-Absorptionsspektralanalyse im Bereich von 200 bis $850 \mathrm{~nm}$, um gelöstes Nitrat und Gesamtnitrat zu bestimmen. Die zweite Multiparametersonde (Exo2 von YSI, Xylem Inc.) kann pH, Temperatur, Leitfähigkeit, Gesamtgehalt gelöster Feststoffe (total dissolved solids, TDS) und Gesamtschwebstoff (total suspended solids, TSS), Trübung und gelösten Sauerstoff bestimmen. Das OnlineMonitoring-System erfasst diese Parameter parallel und in Echtzeit. Ein auf Machine-Learning beruhender Algorithmus wird angewendet, um Vorhersagemodelle für ein übermäßiges Wachstum von Cyanobakterien zu entwickeln. Dazu misst das TRITON-Wasser-Sensorsystem kontinuierlich die Zusammensetzung des Oberflächenwassers. Die gesammelten Daten werden in ein Cloud-basiertes Datenmanagement-System übertragen und in Echtzeit ausgewertet (Abb. 3). Über künstliche Intelligenz werden Vorhersagemodelle für ein übermäßiges Wachstum von Cyanobakterien erstellt. Dazu misst das Sensorsystem kontinuierlich im Oberflächenwasser.

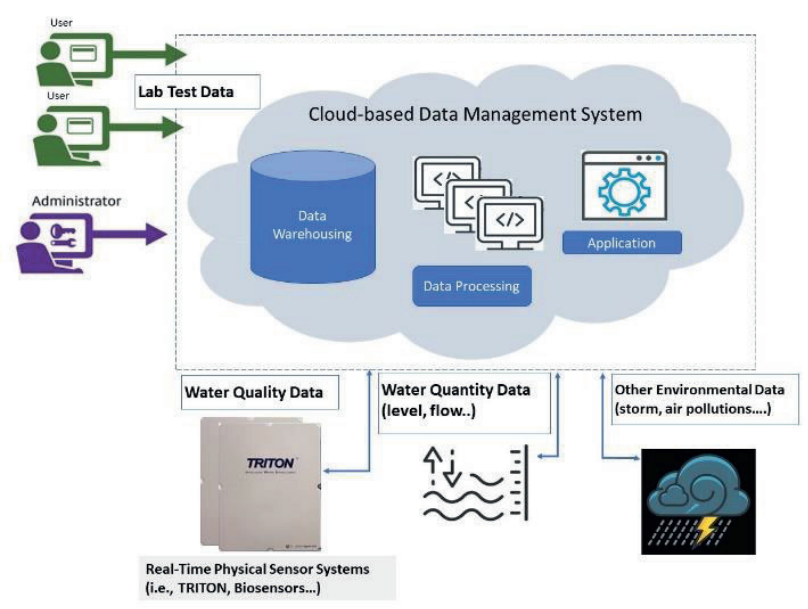

Abb. 3: Cloud-basiertes Datenmanagementsystem für die schnelle Erfassung und Auswertung von Sensordaten, Labortestdaten, Daten zur Erfassung der Wasserquantität und Metadaten aus der Umwelt.

Das physikalisch/chemische Sensorsystem wird für die Anwendung des Lernalgorithmus mit einem Biosensoranalysesystem kombiniert, welches in automatisierter Form Cyanotoxine, wie Microcystin, Anatoxin oder Saxitoxin, vollautomatisch bestimmt. Das
Analyseprinzip beruht auf einen flussbasierten Chemilumineszenz-Mikroarray-Immunoassay. ${ }^{5,6}$ Für die Cyanotoxine wird ein indirekt kompetitiver regenerierbarer Mikroarray-Immunoassay angewendet, der gleichzeitig verschiedene Cyanotoxine innerhalb 7 Minuten quantifizieren kann. Einfach messbare Einflussgrößen werden für ein Vorhersagemodell herangezogen und mit Lerndaten und selektiven $\mathrm{Bi}$ osensordaten abgeglichen. Dies ist wichtig, damit das Vorhersagemodell sich stetig verbessert und schlussendlich die Zielgrößen Algenwachstum und Toxinbildung mit hoher Treffsicherheit vorhergesagt werden können. Damit könnte man ein Frühwarnsystem zur Verfügung stellen, welches Behörden tagesaktuell eine Risikobewertung von Oberflächengewässern ermöglicht.

\section{Diskussion und Ausblick}

Physikalische und chemische Sensoren stehen bereits zur Verfügung. Oftmals sind sie, wie am Beispiel der Exo-Multiparametersonde zu sehen, sehr groß und teuer. Des Weiteren müssen die elektronischen Komponenten in einen klimatisierten Raum gestellt werden und es muss ein Stromanschluss existieren. Sie enthalten aber Vorrichtungen, wie eine kontinuierlich laufende Bürste, damit die Sonde dauerhaft im Oberflächenwasser eingesetzt werden kann. In diesem Sinne ist es eines der ersten Beispiele einer vernetzten Sensorik die im Feld Messdaten erzeugen kann.

Für einige wasseranalytische Fragestellungen existieren mittlerweile erste Sensorkonzepte. ${ }^{7,8}$ Sie müssen jedoch soweit verbessert werden, dass sie feldtauglich werden, robust in komplexen Matrices wie Trinkwasser, Oberflächenwasser, Prozesswasser oder Abwasser mit hoher Präzision quantitative Daten liefern. Des Weiteren müssen sie weiter miniaturisiert werden, damit kostengünstige, mobile und vernetzte Sensorsysteme entstehen, die eine dezentrale Echtzeitanalytik ermöglichen. Durch die intelligente Vernetzung von physikalischen und chemischen Sensoren und das Zusammenführen der Daten auf einem Server können mit Hilfe des Internet-of-Things Monitoringsysteme aufgebaut werden, welche die chemische und hygienische Wasserqualität verbessern. Mit Hilfe von $\mathrm{KI}$ müssen Lernalgorithmen so entwickelt werden, dass die verhältnismäßige unintelligente Sensormessdaten mit Referenzanalytik und Biosensorik von Anfang an trainiert werden. Dies bedeutet, dass bestehende Prozesse nur dann mit Multisensorik und KI gesteuert werden können, wenn viele Messdaten bei definierter Variation des Prozesses generiert werden und diese 
müssen immer wieder mit quantitativen (bio)analytischen Methoden verifiziert werden.

Die Entwicklung einer kostengünstigen anwenderfreundlichen Multisensorik ist Gegenstand der aktuellen Forschung. Die Vernetzung der Sensorsysteme zusammen mit einer Echtzeitdatenübertragung und -auswertung hat ein enormes Potenzial im Rahmen der neuen kabellosen Übertragungswege im 5G-Netz, so dass wir das Internet-of-Things für Frühwarnsysteme zukünftig nutzen können. Biosensorsysteme lassen sich zur schnellen Verifizierung der Wasserqualität einsetzen, indem Spuren von Mikroschadstoffen, Pathogene oder Toxine quantitativ bestimmt werden. Für all diese Anwendungen ist noch viel Forschung notwendig. Aber die Errungenschaften aus der digitalen Welt müssen auf alle Fälle jetzt mit unseren Sensorsystemen integriert werden.

Wenn Sensoren autonom arbeiten, in Echtzeit Daten versenden und diese sogleich intern ausgewertet werden können, kann es zu einem Paradigmenwechsel in der Umweltanalytik führen. Die Wasserqualität kann dezentral sichergestellt werden. Zentrallabore, welche mit aufwändigen instrumentelle Analysesysteme ausgestattet sind, würden nur noch für eine quantitative Bestätigung der Wasserqualität genutzt werden müssen. Hoch komplexe dynamische Prozesse in der Umwelt und in technischen wasserführenden Anlagen könnten autonom geregelt werden, wodurch die Wasserqualität zu jedem Zeitpunkt sichergestellt werden kann. Damit könnten Menschen, Tiere und die Umwelt nachhaltig geschützt werden.

In der Wasserchemische Gesellschaft wurde eine neue Fachgruppe für Sensorik etabliert, die von Dr. Günther Proll vom Institut für Physikalische und Theoretische Chemie an der Eberhard Karls Universität Tübingen geleitet wird. In dieser Fachgruppe, sollen sich nicht nur die Sensoren vernetzen sondern auch die Wassersensoriker.

\section{Literatur}

1. WALSER, S. M.; GERSTNER, D. G.; BRENNER, G.; HÖLLER, C.; LIEBL, B.; HERR, C.W.W: Assessing the environmental health relevance of cooling towers - A systematic review of legionellosis outbreaks. In: Int. J. Hyg. Environ. Health 217 (2016), Nr. 2-3, S. 145-154.

2. CERVERO-ARAGO, S.; SCHRAMMEL, B.; DIETERSDORFER, E.; SOMMER, R.; LÜCK, C.; WALOCHNIK, J.; KIRSCHNER, A.: Viability and infectivity of viable but nonculturable Legionella pneumophila strains induced at high temperatures. In: Water Res. 158 (2019), S. 268-279.

3. LEE, M.W.; HONG, S.H.; CHOU, H.; KIM, J.-H.;
LEE, D.S.; PARK, J.M. Real-time remote monitoring of small-scaled biological wastewater treatment plants by a multivariate statistical process control and neural network-based software sensors. In: Process Biochem. 43 (2008), Nr. 10, S. 1107-1113. 4. SCHMIDT, T.C.: Recent trends in water analysis triggering future monitoring of organic micropollutants. In: Anal. Bioanal. Chem. 410 (2018), S. 39333941.

5. HUEBNER, M.; BEN HADDADA, M.; METHIVIER, C.; NIESSNER, R.; KNOPP, D.; BOUJDAY, S.: Layer-by-layer generation of PEG-based regenerable immunosensing surfaces for small-sized analytes. In: Biosens. Bioelectron. 67 (2015), S. 334-341.

6. SZKOLA, A.; CAMPBELL, K.; ELLIOTT, C.T.; NIESSNER, R.; SEIDEL, M. Automated, high performance, flow-through chemiluminescence microarray for the multiplexed detection of phycotoxins. In: Anal. CHIM. ACTA, 787 (2013), S. 211-218. 7. ZHOU, B.; BIAN, C.; TONG, J.; XIA, S.: Fabrication of a miniature multi-parameter sensor chip for water quality assessment. In: Sensors 17 (2017), Nr. 157, S. 1-14.

8. POSTOLACHE, O.; PEREIRA, J.D.; GIRAO, ET.: Wireless sensor network-based solution for environmental monitoring: water quality assessment case study. In: Sci. Meas. Technol. (2014), S. 1-7.

\section{Danksagung}

Der Autor möchte seinen Fördergebern seinen Dank ausdrücken. Aktuell laufen im Arbeitskreis von PD Dr. Michael Seidel folgende KI-bezogene Projekte: K2I, vom BMBF im Programm Digital Green Tech FONA gefördert; LEMOSE2, vom BMBF im Programm KMU-innovativ gefördert und MARCA, welches von AIF-ZIM gefördert wird. 